\title{
On the frequency variogram and on frequency domain methods for the analysis of spatio-temporal data.
}

\author{
T. Subba Rao \\ University of Manchester,U.K. and \\ C. R. Rao AIMSCS, University of Hyderabad Campus, India \\ Gy. Terdik \\ University of Debrecen, Hungary
}

\begin{abstract}
The covariance function and the variogram play very important roles in modelling and in prediction of spatial and spatio-temporal data. The assumption of second order stationarity, in space and time, is often made in the analysis of spatial data and the spatio-temporal data. Several times the assumption of stationarity is considered to be very restrictive, and therefore, a weaker assumption that the data is Intrinsically stationary both in space and time is often made and used, mainly by the geo-statisticians and other environmental scientists. In this paper we consider the data to be intrinsically stationary. Because of the inclusion of time dimension,the estimation and derivation of the sampling properties of various estimators related to spatio-temporal data become complicated. In this paper our object is to present an alternative way, based on Frequency Domain methods for modelling the data. Here we consider Discrete Fourier Transforms (DFT) defined for the (Intrinsic) time series data observed at several locations as our data, and then consider the estimation of the parameters of spatiotemporal covariance function, estimation of Frequency Variogram, tests of independence etc. We use the well known property that the Discrete Fourier Transforms of stationary time series evaluated at distinct Fourier Frequencies are asymptotically independent and distributed as complex normal in deriving many results considered in this paper. Our object here is to emphasize the usefulness of the Discrete Fourier transforms in the analysis of spatio-temporal data. Under the intrinsic stationarity condition we consider the estimation, discuss the sampling properties of the Frequency Variogram
\end{abstract}


(FV) introduced in an earlier paper by Subba Rao et al. (2014) which was proposed as an alternative to the classical space, time variogram. We show that the FV introduced is a frequency decomposition of the space-time variogram, and can be computed using the Fast Fourier Transform algorithms. Assuming that the DFT's of the intrinsically stationary processes satisfy a Laplacian type of model, an analytic expression for the space-time spectral density function is derived for the intrinsic processes and also an expression for the Frequency Variogram in terms of the spectral density function is also derived. The estimation of the parameters of the spectrum is also considered. A statistical test for spatial independence of spatio-temporal data is also briefly mentioned, and is based on the test proposed earlier by Wahba (1971) for testing independence in multivariate stationary (temporally) time series.

Keywords. Intrinsic stationarity, spatio-temporal random Processes, Frequency Variogram, Laplacian Model, Test for spatial Independence.

Dedication. Professor M. B. Priestley has made many significant contributions to the nonparametric estimation of stationary and nonstationary spectral density functions. He was one of the strong believers of the use of Fourier Transforms, Frequency domain methods in the analysis of time series. This paper is based on the Fourier Transforms and their possible application to spatio-temporal data and is written bearing in mind Professor Priestley's many important contributions in this area. We dedicate this paper to him.

\section{Introduction and Summary}

Spatio temporal data arises in many areas such as agriculture, geology, environmental sciences, finance, etc. Since the data comes from these areas are functions of both time and space, any statistical method developed must take into account both spatial dependence, temporal dependence and any interaction between these two. In the case of spatial data, the second order spatial dependence is measured by the second order covariance function and if the spatial process is second order stationary, then the second order covariance is a function of spatial lag only. In the case of spatio-temporal data the dependence is measured by space-time covariance function and if the process is spatially and temporally stationary, then the covariance function is a function of the spatial lag and temporal lag. These functions are usually estimated under the assumption that the random process is spatially and temporally stationary.

An alternative second order dependence measure is the variogram defined for both spatial processes and spatio-temporal processes. This function is well defined under the weaker assumption of intrinsic stationarity and in view of this it is 
widely used in geo-statistics. Its use is strongly advocated by Cressie (1993), Gringarten and Deutsch (2001) and Sherman (2011) and many others.

If the process is second order stationary, then there is a one to one correspondence between the variogram and the covariance function. The estimation of the spatial covariance, spatial variogram and their asymptotic sampling properties have been considered by several authors Cressie (1993), Yu et al. (2007), Stein (2012), Gneiting et al. (2001), Huang et al. (2011), Gringarten and Deutsch (2001), Ma (2005). The literature on the estimation of space-time covariance function and the space-time variogram is not very extensive in the case of spatiotemporal random processes. The inclusion of temporal dimension complicates the estimation. The estimation and the sampling properties of the spatio- temporal covariance function have been briefly considered by Li et al. (2007), Cressie and Huang (1999), Stein (2005a).

In this paper our objective is to consider the Discrete Fourier Transforms (DFT) of the time series evaluated at Fourier frequencies as our data. If the observed time series data is equally spaced, one can use the Fast Fourier Transform (FFT) algorithm to compute the DFTs. Using the DFT's we model the data. Subba Rao et al. (2014) and Subba Rao and Terdik (2015) use the recently defined 'Frequency variogram ' for the estimation of the parameters of spatiotemporal covariance function of the process assuming that the DFT's satisfy a Complex Stochastic Partial Differential Equation(CSPDE).

We show the spatio temporal variogram and the frequency variogram defined earlier are related. The non-parametric estimation of the frequency variogram is considered. Its sampling properties are discussed. Investigation of the sampling properties of the sample Frequency Variogram is much easier compared to the space-time variogram estimate. We believe that many interesting problems associated with spatio-temporal random processes can be solved using the frequency domain methods. We consider here some of these problems.

We now summarize the contents of the paper. In Section 2, the space time covariance function and space time variogram are introduced, and their estimation, under the assumption of stationarity, is discussed in Section 3. The properties of Discrete Fourier Transforms of stationary spatial processes, spectral representation of the processes are considered in Section 4. The Frequency Variogram and its relation to the classical spatio-temporal variogram, and the non-parametric estimation of the Frequency variogram are considered in Sections 5 and 6 and these are considered under the assumption of Intrinsic stationarity of the process. Assuming that the process is intrinsically stationary, and the intrinsic process satisfies a Laplacian model, an analytic expression for the spectral density of the intrinsic process is obtained in Section 7. The estimation of the parameters of the spectral density function of the Intrinsic process obtained in section 7 , is considered 
in Section 8. The frequency variogram and its relation to the spectral density function is also considered in Section 8. A test for spatial independence, based on the properties of Complex Wishart distribution, is described in Section 9 and the test is based on the test for independence by Wahba (1971).

\section{Space-time Covariance function and the Space-Time Variogram.}

Let $\left\{Y_{t}(\mathbf{s}), \mathbf{s} \in \mathbb{R}^{d}, t \in \mathbb{Z}\right\}$ denote the spatio-temporal random process. Two assumptions are often made which are important for modeling and prediction. They are that the process is second order stationary in space and time and also that the process is isotropic in space. The assumption of stationarity can be sometimes unrealistic. In view of this, another weaker assumption that is often made is that the process is intrinsically stationary. We note that if the process is second order stationary, then it implies that the process is intrinsically stationary. But the converse is not true. We say the process $\left\{Y_{t}(\mathbf{s})\right\}$ is spatially, temporally second order stationary if, for all $t \in Z, \mathbf{s} \in \mathbf{R}^{d}$,

$$
\begin{aligned}
E\left[Y_{t}(\mathbf{s})\right] & =\mu, \\
\operatorname{Var}\left[Y_{t}(\mathbf{s})\right] & =c(0,0)=\sigma_{y}{ }^{2}<\infty, \\
\operatorname{Cov}\left[Y_{t}(\mathbf{s}), Y_{t+u}(\mathbf{s}+\mathbf{h})\right] & =c(\mathbf{h}, u), \mathbf{h} \in \mathbb{R}^{d}, u \in \mathbb{Z} .
\end{aligned}
$$

We note $c(\mathbf{h}, 0)$ and $c(0, u)$ correspond to the purely spatial, purely temporal covariances respectively. Without loss of any generality we assume that $\mu=0$.

The random process is said to be isotropic if

$$
c(\mathbf{h}, u)=c(\|\mathbf{h}\| ; u), \mathbf{h} \in \mathbb{R}^{d}, u \in \mathbb{Z},
$$

where $\|\mathbf{h}\|$ is the Euclidean distance. The process is said to be fully symmetric if $c(\mathbf{h}, u)=c(-\mathbf{h}, u)=c(\mathbf{h},-u)=c(-\mathbf{h},-u)$ (see Gneiting (2002)). The process $\left\{Y_{t}(\mathbf{s})\right\}$ is intrinsically spatially, temporarily stationary if the incremental process, for $u \in Z, \mathbf{h} \in \mathbf{R}^{d}, Y_{t}(\mathbf{s})-Y_{t+u}(\mathbf{s}+\mathbf{h})$ satisfies the following (see Cressie and Wikle (2011),p.315)

$$
\begin{aligned}
E\left(\left(Y_{t}(\mathbf{s})-Y_{t+u}(\mathbf{s}+\mathbf{h})\right)\right. & =0, \\
\operatorname{Var}\left[\left(Y_{t}(\mathbf{s})-Y_{t+u}(\mathbf{s}+\mathbf{h})\right]\right. & =\gamma(\mathbf{h}, u)<\infty . .
\end{aligned}
$$

If $\left\{Y_{t}(\mathbf{s})\right\}$ is isotropic, then

$$
\gamma(\mathbf{h}, u)=\gamma(\|\mathbf{h}\|, u)
$$


where $\gamma(\mathbf{h}, u)$ is also known as the structure function( Yaglom (1987).)

The spatio-temporal variogram is defined as

$$
\gamma(\mathbf{h}, u)=2 \tilde{\gamma}(\mathbf{h}, u)=\operatorname{Var}\left[\left(Y_{t}(\mathbf{s})-Y_{t+u}(\mathbf{s}+\mathbf{h})\right],\right.
$$

and $\tilde{\gamma}(u, \mathbf{h})$ is defined as the semi spatio-temporal variogram. We note that one can define the variogram under the weaker assumption of intrinsic stationarity. In other words we do not need the assumption of stationarity of the original processes. This phenomenon of differencing in space to achieve stationarity is similar to what we have in the case of random processes with stationary increments in time, for instance, the Brownian motion.

Suppose the process $\left\{Y_{t}(\mathbf{s})\right\}$ is spatially and temporally stationary, then we can show

$$
\begin{aligned}
\gamma(\mathbf{h}, u) & =2\left[\operatorname{Var}\left(Y_{t}(\mathbf{s})\right)-\operatorname{Cov}\left(Y_{t}(\mathbf{s}), Y_{t+u}(\mathbf{s}+\mathbf{h})\right)\right] \\
& =2[c(0,0)-c(\mathbf{h}, u)]=2 \tilde{\gamma}(\mathbf{h}, u)
\end{aligned}
$$

and we note that there is a one to one correspondence between $\gamma(\mathbf{h}, u)$ and $c(\mathbf{h}, u)$ in the case of stationary processes. One can show that the covariance function $c(\mathbf{h}, u)$ is positive semi-definite and $\gamma(\mathbf{h}, u)$ is conditionally negative definite.

\section{Estimation of $c(\mathbf{h}, u)$ and $\gamma(\mathbf{h}, u)$.}

Let $\left\{Y_{t}\left(\mathbf{s}_{i}\right) ; i=1,2, \ldots, m ; t=1,2, \ldots, n\right\}$ be a sample from the zero mean, stationary spatio- temporal random process $Y_{t}(\mathbf{s})$. We define the estimates of $c(\mathbf{h}, u)$ and $\gamma(\mathbf{h}, u)$ as follows. (see Sherman (2011) for details). Let

$$
\hat{c}(\mathbf{h}, u)=\frac{1}{|N(\mathbf{h}, u)|} \sum_{N(\mathbf{h}, u)}\left[Y_{t_{i}}\left(\mathbf{s}_{i}\right)-\bar{Y}\left(\mathbf{s}_{i}\right)\right]\left[Y_{t_{j}}\left(\mathbf{s}_{j}\right)-\bar{Y}\left(\mathbf{s}_{j}\right)\right],
$$

where

$$
\bar{Y}\left(\mathbf{s}_{i}\right)=\frac{1}{n} \sum_{t=1}^{n} Y_{t}\left(\mathbf{s}_{i}\right)
$$

and

$$
\hat{\gamma}(\mathbf{h}, u)=\frac{1}{|N(\mathbf{h}, u)|} \sum_{N(\mathbf{h}, u)}\left[Y_{t_{i}}\left(\mathbf{s}_{i}\right)-\left[Y_{t_{j}}\left(\mathbf{s}_{j}\right)\right]^{2},\right.
$$

where $N(\mathbf{h}, u)=\left\{\left(\mathbf{s}_{i}, t_{i}\right),\left(\mathbf{s}_{j}, t_{j}\right) ; \mathbf{s}_{\mathbf{i}}-\mathbf{s}_{\mathbf{j}}=\mathbf{h}\right.$ and $\left.t_{i}-t_{j}=u\right\}$. The estimator $\hat{\gamma}(\mathbf{h}, u)$ is widely known as Matheron estimator. In this paper we are assuming that the time series data observed at all $m$ locations are equally spaced and also there are no missing values. It is interesting to investigate the properties of the estimators proposed here when these assumptions do not hold. 
Under certain conditions, Li et al. (2007) have shown that the sample spatiotemporal covariance function defined above is asymptotically normal.

Based on $\hat{\gamma}(\mathbf{h}, u)$, Cressie (1993) and Huang et al. (2011) have proposed a weighted least squares criterion for estimating the parameters of the theoretical variogram $\gamma(\mathbf{h}, u \mid \theta)$, and Gneiting (2002) proposed a similar criterion for estimating the parameters based on the space- time covariance function $\hat{c}(\mathbf{h}, u)$. Subba Rao et al. (2014) have proposed a frequency domain method for the estimation of the parameters which is robust against departures from Gaussianity and also computationally efficient. The method of estimation proposed by Subba Rao et al. (2014) is similar to Whittle likelihood approach and it is based on the frequency variogram, and the proposed criterion which is easy to compute and is based on Discrete Fourier Transforms. In the following section we define the Frequency Variogram (FV) and derive the sampling properties of the estimator.

\section{Discrete Fourier transforms and the spectral representation of the process $\left\{Y_{t}(\mathbf{s})\right\}$.}

We follow the notation introduced in the paper of Subba Rao and Terdik (2015). Here we briefly highlight and summarize the results we need for our present purposes and for further details we refer to Subba Rao and Terdik (2015) and the books and papers cited in those papers.

We assume the random process $\left\{Y_{t}(\mathbf{s})\right\}$ is second order spatially and temporally stationary. Therefore, the process has the spectral representation given by

$$
Y_{t}(\mathbf{s})=\int_{R^{d}} \int_{-\pi}^{\pi} e^{i(\mathbf{s} \cdot \boldsymbol{\lambda}+t \omega)} d Z_{y}(\boldsymbol{\lambda}, \omega)
$$

where $\mathbf{s} \cdot \boldsymbol{\lambda}=\sum_{i=1}^{d} s_{i} \lambda_{i}$ and $\int_{R^{d}}$, represents a d-fold multiple integral, and $Z_{y}(\boldsymbol{\lambda}, \omega)$ is a zero mean complex valued random process with orthogonal increments and

$$
\begin{aligned}
E\left[d Z_{y}(\boldsymbol{\lambda}, \omega)\right] & =0, \\
E\left|d Z_{y}(\boldsymbol{\lambda}, \omega)\right|^{2} & =d F_{y}(\boldsymbol{\lambda}, \omega),
\end{aligned}
$$

where $d F_{y}(\boldsymbol{\lambda}, \omega)$ is a spectral measure. If we assume further that $d F_{y}(\boldsymbol{\lambda}, \omega)$ is absolutely continuous with respect to Lebesgue measure according to the arguments $\boldsymbol{\lambda}$ and $\omega$, then $d F_{y}(\boldsymbol{\lambda}, \omega)=f_{y}(\boldsymbol{\lambda}, \omega) d \boldsymbol{\lambda} d \omega$, where $d \boldsymbol{\lambda}=\prod_{i=1}^{d} d \lambda_{i}$. Here $f_{y}(\boldsymbol{\lambda}, \omega)$ is a strictly positive, real valued function and is defined as the spatio- temporal spectrum of the random process $\left\{Y_{t}(\mathbf{s})\right\}$, and $-\infty<\lambda_{1}, \lambda_{2}, \ldots, \lambda_{d}<\infty,-\pi \leq \omega \leq \pi$. In view of the orthogonality of the function $Z_{y}(\boldsymbol{\lambda}, \omega)$, it can be shown that

$$
c(\mathbf{h}, u)=\int_{\mathbf{R}^{d}} \int_{-\pi}^{\pi} e^{i(\mathbf{h} \cdot \boldsymbol{\lambda}+u \omega)} f_{y}(\boldsymbol{\lambda}, \omega) d \omega d \boldsymbol{\lambda},
$$


and by inversion we get

$$
f_{y}(\boldsymbol{\lambda}, \omega)=\frac{1}{(2 \pi)^{d+1}} \sum_{u} \int_{-\infty}^{\infty} e^{-i(\mathbf{h} \cdot \boldsymbol{\lambda}+u \omega)} c(\mathbf{h}, u) d \mathbf{h} .
$$

From (1), we have

$$
c(\mathbf{0}, u)=\int_{-\pi}^{\pi} e^{i u \omega} g_{0}(\omega) d \omega
$$

where $g_{0}(\omega)=\int_{-\infty}^{\infty} f_{y}(\boldsymbol{\lambda}, \omega) d \boldsymbol{\lambda}$ is the second order temporal spectral density function of the process $\left\{Y_{t}(\mathbf{s})\right\}$, and in view of our assumption that the process is spatially, temporally stationary $g_{0}(\omega)$ is same for all the locations $\mathbf{s}$. We note $c(\mathbf{h}, u)=c(-\mathbf{h},-u)$ and $f_{y}(\boldsymbol{\lambda}, \omega)=f_{y}(-\boldsymbol{\lambda},-\omega)$, and $f_{y}(\boldsymbol{\lambda}, \omega)>0$ for all $\boldsymbol{\lambda}$ and $\omega$.

Here $\boldsymbol{\lambda}$ is the spatial frequency associated with the spatial coordinates $\mathbf{s}_{i}$ and is usually called the wave number and $\omega$ is the temporal frequency associated with time.

Let $\left\{Y_{t}\left(\mathbf{s}_{\mathbf{i}}\right)\right\} ; i=1,2, \ldots, m ; t=1,2, \ldots n$ be a sample from the zero mean, stationary spatio-temporal random process $\left\{Y_{t}(\mathbf{s})\right\}$. Consider the time series data at the location $\mathbf{s}_{i}$ and define the Discrete Fourier transform (DFT)

$$
J_{\mathbf{s}_{i}}^{y}\left(\omega_{k}\right)=\frac{1}{\sqrt{2 \pi n}} \sum_{t=1}^{n} Y_{t}\left(\mathbf{s}_{i}\right) e^{-i t \omega_{k}} ; \quad(i=1,2, \ldots, \ldots, m)
$$

where $\omega_{k}=\frac{2 \pi k}{n}, k=0,1,2, \ldots,\left[\frac{n}{2}\right]$. We note that the Discrete Fourier transforms can be evaluated using the Fast Fourier Transform (FFT) algorithm(FFT), and the number of operations required to calculate FFT from a time series of length $\mathrm{n}$, is of the order $n(\ln n)$. By inversion, we obtain from (4)

$$
Y_{t}(\mathbf{s})=\sqrt{\frac{n}{2 \pi}} \int_{-\pi}^{\pi} e^{i t \omega} J_{\mathbf{s}}^{y}(\omega) d \omega .
$$

The above representation shows that the $\left\{Y_{t}(\mathbf{s})\right\}$ can be decomposed into sine and cosine terms and the complex valued random variable DFT, $J_{s}^{y}(\omega)$ can be considered as the amplitude corresponding to these sine and cosine basis functions.

We will briefly summarize some well known results associated with DFT's (see Appendix) which will be required later. For details regarding properties of the Discrete Fourier Transforms for stationary processes, we refer to the books of Brillinger (2001) and Giraitis et al. (2012). It is well known that under some structural assumptions (see Giraitis et al. (2012)) the discrete Fourier transforms $\left\{J_{\mathbf{s}}^{y}\left(\omega_{k}\right)\right\}$ evaluated at discrete Fourier frequencies $\omega_{k}$ are asymptotically uncorrelated, and is distributed as complex normal (see for details Brillinger (2001) and Giraitis et al. (2012). 
For example, for large $n$, and for a specific $\omega_{k}$ and for a specific $\mathbf{s},\left\{J_{\mathbf{s}}^{y}\left(\omega_{k}\right)\right\}$ is approximately distributed as complex normal with mean zero and variance $\left\{g_{\mathbf{s}}\left(\omega_{k}\right)\right\}$ which is the second order temporal spectrum of the process at the location $\mathbf{s}$. In view of the spatial stationarity assumption, $g_{\mathbf{s}}\left(\omega_{k}\right)$ is same for all locations, and we denote this common temporal spectrum by $g_{0}\left(\omega_{k}\right)$.

Let $I_{\mathbf{s}}^{y}\left(\omega_{k}\right)=\left|J_{\mathbf{s}}^{y}\left(\omega_{k}\right)\right|^{2}$ be the periodogram, and let $I_{\mathbf{s}_{i}, \mathbf{s}_{j}}^{y}\left(\omega_{k}\right)=J_{\mathbf{s}_{i}}^{y}\left(\omega_{k}\right) J_{\mathbf{s}_{j}}^{y *}\left(\omega_{k}\right)$ be the cross periodogram between the two time series $\left\{Y_{t}\left(\mathbf{s}_{i}\right)\right\}$ and $\left\{Y_{t}\left(\mathbf{s}_{j}\right)\right\}$. In the appendix we summarize some properties of the periodograms (see also Subba Rao and Terdik (2015)). In the following section, we define the Frequency Variogram and consider its estimation and also discuss the asymptotic sampling properties of the estimator proposed.

\section{Frequency Variogram (FV), Properties and its estimation.}

As stated earlier, variogram is used as an alternative measure of second order dependence. It can be defined under weaker conditions and as such it is widely used. Though the statistical properties of the sample variogram are well studied in the case of spatial processes, the estimation and the asymptotic properties of various estimators defined for spatio-temporal processes, such as $\hat{\gamma}(\mathbf{h}, u)$ defined earlier are not well investigated and this could be due to the inclusion of the time dimension in the processes. To circumvent such problems, Subba Rao et al. (2014) have considered frequency domain approach for the statistical analysis, model construction and estimation.

The authors Subba Rao et al. (2014) have introduced frequency variogram as an alternative to spatio-temporal variogram defined earlier and was found to be very useful in the estimation of parameters of spatio-temporal spectrum. As no inversion of high dimensional matrices are required in the estimation suggested, the computation of the minimizing criterion is easy. In this paper we consider further properties of the Frequency Variogram and also discuss its nonparametric estimation. We use the FV as a tool for estimating the parameters of the spatiotemporal spectrum of the intrinsic processes.

Let $\left\{J_{\mathbf{s}}^{y}\left(\omega_{k}\right)\right\}$ be the DFT evaluated at the Fourier frequency $\omega_{k}=\frac{2 \pi k}{n} ; k=$ $0,1,2, \ldots,\left[\frac{n}{2}\right]$ calculated using the time series data $\left\{Y_{t}(\mathbf{s})\right\}$.

The frequency variogram is defined, for a fixed spatial lag $\mathbf{h}$ and at the location $\mathbf{s}$, as follows.

Let

$$
X_{t}^{\mathbf{h}}(\mathbf{s})=Y_{t}(\mathbf{s})-Y_{t}(\mathbf{s}+\mathbf{h}), t=1,2, \ldots n
$$


We have

$$
E\left[X_{t}^{\mathbf{h}}(\mathbf{s})\right]=0, \operatorname{Var}\left[X_{t}^{\mathbf{h}}(\mathbf{s})\right]=\gamma(\mathbf{h}, 0) .
$$

Define the DFT of the time series $\left\{X_{t}^{\mathbf{h}}(\mathbf{s})\right\}$ by

$$
J_{\mathbf{s}, \mathbf{s}+\mathbf{h}}^{x}(\omega)=\frac{1}{\sqrt{2 \pi n}} \sum_{t=1}^{n} X_{t}^{\mathbf{h}}(\mathbf{s}) e^{-i t \omega}=J_{\mathbf{s}}^{y}(\omega)-J_{\mathbf{s}+\mathbf{h}}^{y}(\omega),
$$

and the periodogram by

$$
I_{\mathbf{s}, \mathbf{s}+\mathbf{h}}^{x}(\omega)=\left|J_{\mathbf{s}, \mathbf{s}+\mathbf{h}}^{x}(\omega)\right|^{2} .
$$

\section{Definition 1}

$$
\begin{aligned}
G_{\mathbf{s}, \mathbf{s}+\mathbf{h}}^{x}(\omega) & =2 \widetilde{G}_{\mathbf{s}, \mathbf{s}+\mathbf{h}}^{x}(\omega) \\
& =E\left|J_{\mathbf{s}}^{y}(\omega)-J_{\mathbf{s}+\mathbf{h}}^{y}(\omega)\right|^{2} \\
& =E\left[I_{\mathbf{s}, \mathbf{s}+\mathbf{h}}^{x}(\omega)\right],
\end{aligned}
$$

for all $|\omega| \leq \pi$. Subba Rao et al. (2014) defined $G_{\mathbf{s}, \mathbf{s}+\mathbf{h}}^{x}(\omega)$ as the Frequency Variogram.

We note that $J_{\mathbf{s}, \mathbf{s}+\mathbf{h}}^{x}(\omega)$ is the DFT of the incremental random process $\left\{X_{t}^{\mathbf{h}}(\mathbf{s})\right\}$. If the incremental process defined is spatially intrinsically stationary, and also temporally stationary, then the discrete Fourier transforms $\left\{J_{\mathbf{s}, \mathbf{s} \pm \mathbf{b}}^{x}\left(\omega_{k}\right)\right\}$ are asymptotically uncorrelated, and distributed as Complex Gaussian (Brillinger (2001) and Giraitis et al. (2012) ). These functions are well defined and no assumptions of spatial, temporal stationarity of the process $\left\{Y_{t}(\mathbf{s})\right\}$ is required. The FV $G_{\mathbf{s}, \mathbf{s}+\mathbf{h}}^{x}(\omega)$ can be used as a measure of dissimilarity between the two random process $\left\{Y_{t}(\mathbf{s})\right\}$ and $\left\{Y_{t}(\mathbf{s}+\mathbf{h})\right\}$ at the frequency $\omega$. As one would expect this measure to increase as the spatial lag $\|\mathbf{h}\|$ increases and tends to zero as $\|\mathbf{h}\| \rightarrow \mathbf{0}$. Some further comments on FV are in order.

Remark 1 The FV given by $G_{\mathbf{s}, \mathbf{s}+\mathbf{h}}^{x}(\omega)$ is well defined and defined under the weaker condition of Intrinsic stationarity.

Remark 2 If the intrinsic process $\left\{X_{t}^{\mathbf{h}}(\mathbf{s})\right\}$ is spatially and temporally stationary, its second order periodogram $I_{\mathbf{s}, \mathbf{s}+\mathbf{h}}^{x}(\omega)$ is asymptotically an unbiased estimator of the temporal spectrum of the intrinsic process $\left\{X_{t}^{\mathbf{h}}(\mathbf{s})\right\}$. In view of the assumption of the spatial stationarity of the intrinsic process, the second order spectrum does not depend on the location $\mathbf{s}$. Therefore estimating the FV is same as estimating the second order spectral density function of the intrinsic process $\left\{X_{t}^{\mathbf{h}}(\mathbf{s})\right\}$. This estimation is considered in section 6 . 
In the following we show the relationship between the spatio temporal variogram $\gamma(\mathbf{h}, u)$ and the FV.

Proposition 1 Let

$$
G_{\mathbf{s}, \mathbf{s}+\mathbf{h}}^{x}(\omega)=E\left|J_{\mathbf{s}, \mathbf{s}+\mathbf{h}}^{x}(\omega)\right|^{2}
$$

then

$$
\int_{-\pi}^{\pi} G_{\mathbf{s}, \mathbf{s}+\mathbf{h}}^{x}(\omega) d \omega=\gamma(\mathbf{h}, 0)
$$

Proof. An application of Parseval's theorem gives the above result.

In the derivation of the above we used the assumption that the incremental process $\left\{X_{t}^{\mathbf{h}}(\mathbf{s})\right\}$ is stationary temporally and spatially even though the original process $\left\{Y_{t}(\mathbf{s})\right\}$ may not be spatially, temporally stationary.

The above result ([6) shows that the FV, $G_{\mathbf{s}, \mathbf{s}+\mathbf{h}}^{x}(\omega)$ is the frequency decomposition of the classical spatio temporal variogram $\gamma(\mathbf{h}, u)$ when $u=0$, similar to the frequency decomposition we have for the power (variance) of the stationary random process in terms of the power spectral density function. Since $\gamma(\mathbf{h}, u)$ is a measure of dissimilarity between two spatial processes separated by lag $\mathbf{h}$, $G_{\mathbf{s}, \mathbf{s}+\mathbf{h}}^{x}(\omega)$ is also a measure of dissimilarity of the two process at the frequency $\omega$. By plotting this function as a function of $\omega$, one can observe in which frequency band there is a large amount of lack of similarity. This information could be useful in prediction where one can predict a time series using the time series data from other neighborhood locations.

Proposition 2 . Let $\left\{Y_{t}(\mathbf{s})\right\}$ be a zero-mean second order stationary process in space and time and let $\left\{J_{\mathbf{s}_{i}}^{y}(\omega)\right\}(i=1,2, \ldots, m)$ be the DFT's of $\left\{Y_{t}\left(\mathbf{s}_{i}\right), i=1,2, \ldots m\right\}$. Let $G_{\mathbf{s}_{i}, \mathbf{s}_{j}}^{x}(\omega)$ be the frequency variogram. Then

1. The covariance function $g_{\mathbf{s}_{i}, \mathbf{s}_{j}}^{y}(\omega)=\operatorname{cov}\left(J_{\mathbf{s}_{i}}^{y}(\omega), J_{\mathbf{s}_{j}}^{x}(\omega)\right)$ is a positive semidefinite function.

2. The $F V G_{\mathbf{s}_{i}, \mathbf{s}_{j}}^{x}(\omega)$ is conditionally negative definite.

Proof. Consider the sum $S_{1}(\omega)=\sum_{i=1}^{m} a_{i} J_{\mathbf{s}_{i}}^{y}(\omega)$ where $\left\{a_{i}\right\}$ can be complex. Then

$$
\operatorname{Var} S_{1}(\omega)=\sum \sum a_{i} a_{j}^{*} \operatorname{Cov}\left(J_{\mathbf{s}_{i}}^{y}(\omega), J_{\mathbf{s}_{j}}^{y}(\omega)\right) \geq 0
$$

Hence the result (1).

To prove the second result, assume $\sum a_{i}=0$. Then we can show that

$$
\begin{aligned}
\sum \sum a_{i} a_{j}^{*} G_{\mathbf{s}_{i}, \mathbf{s}_{j}}^{x}(\omega) & =\sum \sum a_{i} a_{j}^{*} E\left|J_{\mathbf{s}_{i}}^{y}(\omega)-J_{\mathbf{s}_{j}}^{y}(\omega)\right|^{2} \\
& =-2 \operatorname{Var}\left[\sum_{i=1}^{m} a_{i} J_{\mathbf{s}_{i}}^{y}(\omega)\right] \leq 0 .
\end{aligned}
$$


In the above derivation we used the fact that $\sum a_{i}=0$ and also the second order spectral density function does not depend on the location $\mathbf{s}_{i}$ because of stationarity assumption. Hence the result (2) of the proposition.

\subsection{Frequency Variogram and Nugget Effect:}

For illustration purposes we consider the case $d=2$. Suppose instead of observing the process $\left\{Y_{t}(\mathbf{s}), \mathbf{s} \in \mathbb{R}^{2}, t \in \mathbb{Z}\right\}$, we observe a corrupted random process $\left\{\widetilde{Y}_{t}(\mathbf{s}), \mathbf{s} \in \mathbb{R}^{2}, t \in \mathbb{Z}\right\}$, where for each $\mathbf{s}$ and $t$

$$
\widetilde{Y}_{t}(\mathbf{s})=Y_{t}(\mathbf{s})+\eta_{t}(\mathbf{s})
$$

and $\left\{Y_{t}(\mathbf{s})\right\}$ and $\left\{\eta_{t}(\mathbf{s})\right\}$ are zero mean spatially, temporally stationary processes and $\left\{Y_{t}(\mathbf{s})\right\}$ and $\left\{\eta_{t}(\mathbf{s})\right\}$ are independent for all $t$ and $\mathbf{s}$, it is defined as a generalized process. Further, we assume that $\left\{\eta_{t}(\mathbf{s})\right\}$ is a white noise process in space and time with the second order space-time spectrum $g_{\eta}(\boldsymbol{\lambda}, \omega)=\frac{\sigma_{\eta}{ }^{2}}{(2 \pi)^{3}}$ for all $\boldsymbol{\lambda}$ and $\omega$. Define the DFT of the incremental random process of $\left\{\widetilde{Y}_{t}(\mathbf{s})\right\}$,

$$
\left(\widetilde{Y}_{t}(\mathbf{s})-\tilde{Y}_{t}(\mathbf{s}+\mathbf{h})\right)=\left(Y_{t}(\mathbf{s})-Y_{t}(\mathbf{s}+\mathbf{h})\right)+\left(\eta_{t}(\mathbf{s})-\eta_{t}(\mathbf{s}+\mathbf{h}),\right.
$$

then we have

$$
\widetilde{J}_{\mathbf{s}, \mathbf{s}+\mathbf{h}}(\omega)=J_{\mathbf{s}, \mathbf{s}+\mathbf{h}}^{x}(\omega)+J_{\mathbf{s}, \mathbf{s}+\mathbf{h}}^{\eta}(\omega), \quad|\omega| \leq \pi,
$$

where

$$
\begin{aligned}
& \widetilde{J}_{\mathbf{s}, \mathbf{s}+\mathbf{h}}(\omega)=\frac{1}{\sqrt{2 \pi n}} \sum\left(\widetilde{Y}_{t}(\mathbf{s})-\widetilde{Y}_{t}(\mathbf{s}+\mathbf{h})\right) e^{-i \omega t}, \\
& J_{\mathbf{s}, \mathbf{s}+\mathbf{h}}^{x}(\omega)=\frac{1}{\sqrt{2 \pi n}} \sum\left(Y_{t}(\mathbf{s})-Y_{t}(\mathbf{s}+\mathbf{h})\right) e^{-i \omega t}, \\
& J_{\mathbf{s}, \mathbf{s}+\mathbf{h}}^{\eta}(\omega)=\frac{1}{\sqrt{2 \pi n}} \sum\left(\eta_{t}(\mathbf{s})-\eta_{t}(\mathbf{s}+\mathbf{h})\right) e^{-i \omega t} .
\end{aligned}
$$

Define the FV for the process $\left\{\tilde{Y}_{t}(\mathbf{s})\right\}$,

$$
\begin{aligned}
\widetilde{G}_{\mathbf{s}, \mathbf{s}+\mathbf{h}}(\omega) & =E\left|\widetilde{J}_{\mathbf{s}, \mathbf{s}+\mathbf{h}}(\omega)\right|^{2} \\
& =E\left|J^{x}{ }_{\mathbf{s}, \mathbf{s}+\mathbf{h}}(\omega)\right|^{2}+E\left|J_{\mathbf{s}, \mathbf{s}+\mathbf{h}}^{\eta}(\omega)\right|^{2} \\
& =G_{\mathbf{s}, \mathbf{s}+\mathbf{h}}^{x}(\omega)+\frac{2 \sigma_{\eta}^{2}}{(2 \pi)^{3}} .
\end{aligned}
$$


The above result follows because of our assumption that the random process $\left\{\eta_{t}(\mathbf{s})\right\}$ is a white noise. From (17), we observe that as $\|\mathbf{h}\| \rightarrow 0, G_{\mathbf{s}, \mathbf{s}+\mathbf{h}}^{x}(\omega) \rightarrow 0$ for all $\omega$ and, therefore, $\widetilde{G}_{\mathbf{s}, \mathbf{s}+\mathbf{h}}(\omega) \rightarrow \frac{\sigma_{\eta}^{2}}{(2 \pi)^{3}}$ as $\|\mathbf{h}\| \rightarrow 0$.

If we plot $\int G_{\mathbf{s , s} \mathbf{s}}(\omega) d \omega$ as a function of $\|\mathbf{h}\|$ and if we observe a jump near the origin $\|\mathbf{h}\|=0$, this could be due to the presence of white noise in the process. In other words, the observations are corrupted by white noise. This effect is usually called the "Nugget effect" in geo-mining literature. In the following section we consider the estimation of $G_{\mathbf{s}, \mathbf{s}+\mathbf{h}}(\omega)$ when the observations are not corrupted. In practice one uses the Fast Fourier Transform algorithm for computing the DFT's when the time series data is equally spaced.

We may point out that other types of Nugget effects are feasible, for example one could have a process which is temporally correlated, but spatially uncorrelated. Such processes were discussed by Stein (2005b).

\section{Estimation of the Frequency variogram under the Intrinsic stationarity.}

Let $\left\{Y_{t}\left(\mathbf{s}_{i}\right) ; i=1,2,3, \ldots, m ; t=1,2, \ldots, n\right\}$ be a sample from the spatio temporal random process $\left\{Y_{t}\left(\mathbf{s}_{i}\right)\right\}$. Here we consider the estimation of $\mathrm{FV}$ under the assumption that the process is intrinsically stationary both spatially and temporally. We assume that the process $\left\{Y_{t}\left(\mathbf{s}_{i}\right)\right\}$ observed is not corrupted by noise.

Consider the Frequency Variogram $G_{\mathbf{s}, \mathbf{s}+\mathbf{h}}^{x}(\omega)=E\left|J_{\mathbf{s}}^{y}(\omega)-J_{\mathbf{s}+\mathbf{h}}^{y}(\omega)\right|^{2},|\omega| \leq$ $\pi$. We noted earlier that the $\mathrm{FV} G_{\mathbf{s}, \mathbf{s}+\mathbf{h}}^{x}(\omega)$ is the expected value of the periodogram of the incremental process $X_{t}^{\mathbf{h}}(\mathbf{s})=Y_{t}(\mathbf{s})-Y_{t}(\mathbf{s}+\mathbf{h}),(t=1,2, \ldots)$. The $\operatorname{process}\left\{X_{t}^{\mathbf{h}}(\mathbf{s})\right\}$ is spatially, temporally stationary when $\mathbf{h}$ is fixed. Therefore for large $n$, it is well known that the periodogram is an unbiased estimator of the second order spectral density function of the stationary process $\left\{X_{t}^{\mathbf{h}}(\mathbf{s})\right\}$ though it is not a consistent estimator. Therefore, our object here is to obtain a consistent estimator of the spectrum of the incremental process $\left\{X_{t}^{\mathbf{h}}(\mathbf{s})\right\}$ for a given $\mathbf{h}$, using the entire sample of discrete of Fourier transforms $\left\{J_{\mathbf{s}_{i}}\left(\omega_{k}\right) ; i=1,2, \ldots, m\right\}$, for all $\omega_{k}=\frac{2 \pi k}{n},\left(k=0,1, \ldots,\left[\frac{n}{2}\right]\right)$.

Let $g_{\mathbf{s}_{i}, h}^{x}(\omega)$ be the second order spectrum of the incremental process $\left\{X_{t}^{\mathbf{h}}\left(\mathbf{s}_{i}\right)\right\}$. Since the intrinsic process is spatially stationary $g_{\mathbf{s}_{i} \mathbf{h}}^{x}(\omega)$ does not depend $\mathbf{s}_{i}$. We denote such a stationary spectrum of the intrinsic process by $g_{\mathbf{h}}^{x}(\omega)$.

Let $\Omega$ denote the set of all location $\mathbf{s}_{1}, \mathbf{s}_{2}, \ldots \mathbf{s}_{m}$, and let $N(\mathbf{h})$ denote the subset of locations, such that $N(\mathbf{h})=\left\{\mathbf{s}_{i} ; i=1,2, \ldots, m\right.$, such that, both $\left.\mathbf{s}_{i}, \mathbf{s}_{i}+\mathbf{h} \in \Omega\right\}$, $|N(\mathbf{h})|$ be the number of distinct elements in the set $N(\mathbf{h})$. The estimation of stationary spectrum of a time series is well known and, therefore, we discuss the estimation of $g_{\mathbf{h}}^{x}(\omega)$ only briefly. For details, we refer to Priestley (1981), Brillinger 
(2001), Brockwell and Davis (1987).

Consider the estimator,

$$
\hat{g}_{\mathbf{h}}^{x}(\omega)=\int_{-\pi}^{\pi} W_{n}(\omega-\theta)\left(\frac{1}{|N(\mathbf{h})|} \sum_{i} I_{\mathbf{s}_{i}, \mathbf{s}_{i}+\mathbf{h}}^{x}(\theta) d \theta\right),
$$

where the sum is taken over the set $N(\mathbf{h})$, and the weight function $W_{n}(\theta)$, which is a real valued even function of $\theta$, satisfies the following Assumptions. For further details, see (Priestley (1981), Brillinger (2001)).

\section{Assumptions:}

1. $W_{n}(\theta) \geq 0$ for all $n$ and $\theta$,

2. $\int W_{n}(\theta) d \theta=1$, all $n$,

3. $\int W_{n}^{2}(\theta) d \theta<\infty$, all $n$,

4. For any $\varepsilon(>0), W_{n}(\theta) \rightarrow 0$, uniformly as $n \rightarrow \infty$, for $|\theta|>\varepsilon$.

Theorem 1 Let $g_{\mathbf{h}}^{x}(\omega)$ be the spectral density function of the process $\left\{X_{t}^{\mathbf{h}}\left(\mathbf{s}_{i}\right)\right\}$ for all $\mathbf{s}_{i}$ and let $g_{\mathbf{s}_{i}, \mathbf{s}_{j}}^{x}(\mathbf{h}, \omega)$ be the cross spectral density function of the process $\left\{X_{t}^{\mathbf{h}}\left(\mathbf{s}_{i}\right)\right\}$ and $\left\{X_{t}^{\mathbf{h}}\left(\mathbf{s}_{j}\right)\right\}$. Then we have

$$
E\left(\hat{g}_{\mathbf{h}}^{x}(\omega)\right)=g_{\mathbf{h}}^{x}(\omega)+O\left(\frac{\ln n}{n}\right),
$$

and

$$
\lim _{n \rightarrow \infty} \operatorname{Var}\left(\hat{g}_{h}(\omega)\right)=\frac{1}{|N(\mathbf{h})|^{2}} \frac{2 \pi}{n} \int W_{n}^{2}(\omega-\theta)\left[\sum_{i, j}\left|g_{\mathbf{s}_{i}, \mathbf{s}_{j}}^{x}(\mathbf{h}, \theta)\right|^{2}\right] d \theta .
$$

Proof. Take expectations both sides of (8)

$$
E\left(\hat{g}_{h}^{x}(\omega)\right)=\int W_{n}(\omega-\theta)\left(\frac{1}{|N(\mathbf{h})|}\right) \sum_{i} E\left(I_{\mathbf{s}_{i}, \mathbf{s}_{i}+\mathbf{h}}^{x}(\theta)\right) d \theta,
$$

and we have

$$
E\left(I_{\mathbf{s}_{i}, \mathbf{s}_{i}+\mathbf{h}}^{x}(\theta)\right)=g_{\mathbf{h}}^{x}(\theta)+O\left(\frac{\ln n}{n}\right),
$$

and, therefore, we obtain

$$
E\left(\hat{g}_{\mathbf{h}}^{x}(\omega)\right)=g_{\mathbf{h}}^{x}(\omega)+O\left(\frac{\ln n}{n}\right),
$$


in view of the Assumption 2, and the fact that $W_{n}(\theta)$ is approaching the DiracDelta function concentrating its mass at $\theta=0$. Therefore, $\hat{g}_{\mathbf{h}}^{x}(\omega)$ is asymptotically an unbiased estimator of $g_{\mathbf{h}}^{x}(\omega)$. As we have noted earlier, estimating the frequency variogram is equivalent to (for large $n$ ) estimating the spectral density $g_{\mathbf{h}}^{x}(\omega)$ of the intrinsic process $\left\{X_{t}^{\mathbf{h}}\left(\mathbf{s}_{i}\right)\right\}$. To obtain an expression for the variance, we consider a discrete approximation of $\widehat{g}_{\mathbf{h}}^{x}(\omega)$. Our derivation here is heuristic, and to obtain an expression for the covariance we assume the intrinsic process is Gaussian, even though this assumption is not essential for proving normality or consistency (see Brillinger (2001), and Giraitis et al. (2012)). Consider the discrete approximation of (8), and take variance both sides, we get

$$
\begin{aligned}
\operatorname{Var}\left(\hat{g}_{\mathbf{h}}^{x}(\omega)=\frac{1}{|N(\mathbf{h})|^{2}}\left(\frac{2 \pi}{n}\right)^{2} \sum_{P}\right. & \sum_{P^{\prime}} W_{n}\left(\omega-\theta_{P}\right) W_{n}\left(\omega-\theta_{P^{\prime}}\right) \\
& \times \operatorname{Cov}\left(\sum_{i} I_{\mathbf{s}_{i}, \mathbf{s}_{i}+\mathbf{h}}^{x}\left(\theta_{P}\right), \sum_{j} I_{\mathbf{s}_{j}, \mathbf{s}_{j+\mathbf{h}}}^{x}\left(\theta_{P^{\prime}}\right)\right),
\end{aligned}
$$

and we have

$$
\begin{aligned}
& \operatorname{Cov}\left(\sum_{i} I_{\mathbf{s}_{i}, \mathbf{s}_{i}+\mathbf{h}}^{x}\left(\theta_{P}\right), \sum_{j} I_{\mathbf{s}_{j}, \mathbf{s}_{j}+\mathbf{h}}^{x}\left(\theta_{P^{\prime}}\right)\right) \\
& =\eta\left(\theta_{p}-\theta_{p^{\prime}}\right) \sum_{i} \sum_{j}\left|g_{\mathbf{s}_{i}, \mathbf{s}_{j}}^{x}\left(\mathbf{h}, \theta_{p}\right)\right|^{2}+\eta\left(\theta_{p}+\theta_{p^{\prime}}\right) \sum_{i} \sum_{j}\left|g_{\mathbf{s}_{i}, \mathbf{s}_{j}}^{x}\left(\mathbf{h}, \theta_{p}\right)\right|^{2},
\end{aligned}
$$

where $\eta(\theta)=\sum_{-\infty}^{\infty} \delta(\theta-2 \pi j)$ is a Dirac comb (Brillinger (2001) Corollary 7.22). To obtain the above expression we used the results already well known concerning the covariance between two periodogram ordinates (see Brillinger (2001)).After substitution of this expression for the covariance and after some simplification, we obtain

$$
\left.\lim _{n \rightarrow \infty} \operatorname{Var}\left(\hat{g}_{\mathbf{h}}(\omega)\right)=\frac{1}{|N(\mathbf{h})|^{2}} \frac{2 \pi}{n} \int W_{n}^{2}(\omega-\theta)\left[\sum \sum\left(g_{\mathbf{s}_{i}, \mathbf{s}_{j}}^{x}(\mathbf{h}, \theta)\right)^{2}\right)\right] d \theta .
$$

The above result shows that $\hat{g}_{h}^{x}(\omega)$ is a mean square consistent estimator of $g_{h}^{x}(\omega)$ and as we mentioned earlier that $g_{h}^{x}(\omega)$ is asymptotically equivalent to the Frequency Variogram.

Remark 3 In the derivation of the above results, we have only assumed that the intrinsic process is Gaussian. The assumption of Gaussianity is made only to obtain a simple expression for the variance. The result that the estimator $\widehat{q}_{\mathbf{b}}^{x}(\omega)$ is a consistent estimator is still valid under non Gaussianity assumption (see Brillinger (2001)). 
Remark 4 It is well known that the usual Matheron estimator for the Variogram $\gamma(\mathbf{h}, \mathbf{u})$ may not be stable if the data are sparse or irregularly shaped (see Schabenberger and Gotway (2005),p 153). In such situations, it is usual to consider all pairs $\left(\mathbf{s}_{i}, \mathbf{s}_{j}\right)$ such that $\mathbf{s}_{i}-\mathbf{s}_{j}=\mathbf{h} \pm \triangle$, where $\triangle$ is tolerance (see Cressie and Huang (1999)). The choice of $\triangle$ is arbitrary and the derivation of the sampling properties become complicated. (1981))

We can show by following similar lines as above, that as $n \rightarrow \infty$, (see Priestley

$$
\operatorname{Cov}\left(\hat{g}_{\mathbf{h}}^{x}\left(\omega_{1}\right), \hat{g}_{\mathbf{h}}^{x}\left(\omega_{2}\right)\right)=0 \quad \text { for } \quad \omega_{1}+\omega_{2} \neq 0 .
$$

The asymptotic normality of $\hat{g}_{\mathbf{b}}^{x}(\omega)$ can be shown using the results of Hannan (1973), Taniguchi (1980), Deo and Chen (2000).

\section{Complex Stochastic Partial Differential Equa- tion(CSPDE) for the intrinsic process and the spectrum for the FV.}

In a recent paper, Subba Rao and Terdik (2015) defined a complex stochastic partial differential equation for the spatio temporal process and obtained an analytic expression for the spectrum of the spatio-temporal process. The parametric spectrum thus obtained from the assumed model is non-separable.A spatio-temporal random process is said to be separable if its second order space-time spectrum can be written as a product of two positive semi-definte functions which are ,in fact, space spectrum which is a function of wave numbers $\lambda$, and the other part corresponds to temporal spectrum corresponding to the temporal frequency $\omega$. As we mentioned earlier, stationarity assumption may not be realistic always, and therefore, a weaker assumption that the process is intrinsically stationary is made. Here our object is to define a model for such an intrinsic process, and obtain an analytic parametric expression for the spectrum for the intrinsic process. In a later section, we consider the estimation of the parameters of the spectral function. We may note that Yaglom (1987) and Huang et al. (2011) and others have obtained spectra for the variogram in the case of spatial process. Yu et al. (2007), Huang et al. (2011), have considered non-parametric estimation of the variogram.

Consider the incremental random process $X_{t}^{\mathbf{h}}(s)=Y_{t}(\mathbf{s})-Y_{t}(\mathbf{s}+\mathbf{h}), \mathbf{s} \in \mathbb{R}^{d}, t \in$ $\mathbb{Z}$. For a fixed $\mathbf{h}$, the incremental process is a function of the spatial location $\mathbf{s} \in \mathbb{R}^{d}$, and time $t \in \mathbb{Z}$

We consider the process $\left\{X_{t}^{\mathbf{h}}(s)\right\}$ which is assumed to be a zero mean, station- 
ary process in space and time. Define the DFT of the time series $\left\{X_{t}^{\mathbf{h}}(s)\right\}$,

$$
J_{\mathbf{s}, \mathbf{s}+\mathbf{h}}^{(x)}\left(\omega_{k}\right)=\frac{1}{\sqrt{2 \pi n}} \sum_{t=1}^{n} X_{t}^{\mathbf{h}}(s) e^{i t \omega_{k}},
$$

and the $\operatorname{DFT} J_{\mathbf{s}(\mathbf{L}), \mathbf{s}(\mathbf{L})+\mathbf{h}}^{(x)}\left(\omega_{k}\right)$ of the time series $\left\{X_{t}^{\mathbf{h}}(\mathbf{s}(\mathbf{L}))\right\}$ where, for each $t, X_{t}^{\mathbf{h}}(\mathbf{s}(\mathbf{L}))=$ $Y_{t}(\mathbf{s}+\mathbf{L})-Y_{t}(\mathbf{s}+\mathbf{L}+\mathbf{h})$ at the frequencies

$$
\omega_{k}=\frac{2 \pi k}{n},\left(k=0,1,2, \ldots,\left[\frac{n}{2}\right]\right) .
$$

Define the covariance between two distinct Fourier Transforms $J_{\mathbf{s}, \mathbf{s}+\mathbf{h}}^{(x)}(\omega)$ and $J_{\mathbf{s}(\mathbf{L}), \mathbf{s}(\mathbf{L})+\mathbf{h}}^{(x)}(\omega)$,

$$
g_{\mathbf{s}, \mathbf{s}+\mathbf{L}}^{(\mathbf{h})}(\omega)=\operatorname{Cov}\left(J_{\mathbf{s}, \mathbf{s}+\mathbf{h}}^{(x)}(\omega), J_{\mathbf{s}(\mathbf{L}) \mathbf{s}(\mathbf{L})+\mathbf{h}}^{(x)}(\omega)\right),
$$

where $J_{\mathbf{s}, \mathbf{s}+\mathbf{h}}^{(x)}(\omega), J_{\mathbf{s}(\mathbf{L}), \mathbf{s}(\mathbf{L})+\mathbf{h}}^{(x)}(\omega)$, respectively, are discrete Fourier Transforms of the incremental processes

$$
X_{t}^{\mathbf{h}}(s)=Y_{t}(\mathbf{s})-Y_{t}(\mathbf{s}+\mathbf{h}), \quad \text { and } \quad X_{t}^{\mathbf{h}}(\mathbf{s}(\mathbf{L}))=Y_{t}(\mathbf{s}+\mathbf{L})-Y_{t}(\mathbf{s}+\mathbf{L}+\mathbf{h}),
$$

for $t=1, \ldots, n, \quad \mathbf{s}=\mathbf{s}_{1}, \ldots, \mathbf{s}_{m}$ and $\mathbf{L} \in \mathbf{R}^{d}$. We note that in computing the above, we fix $\mathbf{h}$ and consider $\left\{X_{t}^{\mathbf{h}}(\mathbf{s})\right\}$ as one spatio-temporal series.

Since the process $\left\{X_{t}^{\mathbf{h}}(\mathbf{s})\right\}$ is a zero mean second order spatially, temporally stationary, it has the spectral representation.

$$
X_{t}^{\mathbf{h}}(\mathbf{s})=\int_{\mathbf{R}^{d}} \int_{-\pi}^{\pi} e^{i(\mathbf{s} \cdot \boldsymbol{\lambda}+t \omega)} d \xi_{X}^{(\mathbf{h})}(\boldsymbol{\lambda}, \omega) .
$$

where $d \xi_{X}^{(\mathbf{h})}(\boldsymbol{\lambda}, \omega)$ is a zero mean complex random process with orthogonal increments with

$$
\begin{aligned}
E\left[d \xi_{X}^{(\mathbf{h})}(\boldsymbol{\lambda}, \omega)\right] & =0, \\
E\left|d \xi_{X}^{(\mathbf{h})}(\boldsymbol{\lambda}, \omega)\right|^{2} & =d F_{X}{ }^{(\mathbf{h})}(\boldsymbol{\lambda}, \omega)=f_{X}^{(\mathbf{h})}(\boldsymbol{\lambda}, \omega) d \boldsymbol{\lambda} d \omega .
\end{aligned}
$$

We define $f_{X}{ }^{(\mathbf{h})}(\boldsymbol{\lambda}, \omega)$, as the spectral density function of the stationary intrinsic process $\left\{X_{t}^{\mathbf{h}}(\mathbf{s})\right\}$. We have the following spectral representation for the DFT of the intrinsic process.

Proposition 3 Let $J_{\mathbf{s}, \mathbf{s}+\mathbf{h}}^{(x)}(\omega)$ be the DFT of the stationary time series $\left\{X_{t}^{\mathbf{h}}(\mathbf{s})\right\}$. Then,

$$
J_{\mathbf{s}, \mathbf{s}+\mathbf{h}}^{(x)}(\omega)=\sqrt{\frac{n}{2 \pi}} \int e^{i \mathbf{s} \cdot \boldsymbol{\lambda}} d \xi_{X}{ }^{(\mathbf{h})}(\boldsymbol{\lambda}, \omega)+o_{p}(1) .
$$


Proof. The proof is similar to the proof given in Proposition 2 of Subba Rao and Terdik (2015) and hence the details are omitted.

In the following we denote the $d$ coordinates of the location $\mathbf{s}$ by $\left(s_{1}, s_{2} \ldots s_{d}\right)$.

Theorem 2 Let $\left\{J_{\mathbf{s}_{i}, \mathbf{s}_{i}+\mathbf{h}}^{(x)}(\omega) ; i=1,2, \ldots, m\right\}$ be the discrete Fourier transforms of the incremental process $\left\{X_{t}^{\mathbf{h}}\left(\mathbf{s}_{i}\right)\right\}$. Let

$$
\left[\sum_{i=1}^{d} \frac{\partial^{2}}{\partial s_{i}{ }^{2}}-\left|P_{\mathbf{h}}(\omega, \boldsymbol{\psi})\right|^{2}\right]^{\nu} J_{\mathbf{s}, \mathbf{s}+\mathbf{h}}^{(x)}(\omega)=J_{\eta_{\mathbf{s}}}^{(\mathbf{h})}(\omega), \quad|\omega| \leq \pi,
$$

where $\nu>0$, and $J_{\eta_{\mathbf{s}}}^{(\mathbf{h})}(\omega)$ is the DFT of the space-time white noise process $\left\{\eta_{t}(\mathbf{s})\right\}$ and $P_{\mathbf{h}}(\omega, \boldsymbol{\psi})$ is a polynomial in $\omega$ and it is a function of some parameter vector $\boldsymbol{\psi}$. Then the second order space-time spectrum of the intrinsic process $\left\{X_{t}^{\mathbf{h}}(\mathbf{s})\right\}$ is given by

$$
f_{X}^{(\mathbf{h})}(\boldsymbol{\lambda}, \omega)=\frac{\sigma_{\eta}{ }^{2}}{(2 \pi)^{d+1}} \frac{1}{\left(\sum_{i=1}^{d} \lambda_{i}^{2}+\left|P_{\mathbf{h}}(\omega, \boldsymbol{\psi})\right|^{2}\right)^{2 \nu}},
$$

and the covariance between the periodograms (which is a spectrum dependent on spatial distance $\mathbf{L}$, and the temporal frequency $\omega$ ) is given by

$$
\begin{aligned}
g_{\mathbf{s}, \mathbf{s}+\mathbf{L}}^{(\mathbf{h})}(\omega) & =\operatorname{Cov}\left(J_{\mathbf{s}, \mathbf{s}+\mathbf{h}}^{(x)}(\omega), J_{\mathbf{s}(\mathbf{L}), \mathbf{s}(\mathbf{L})+\mathbf{h}}^{(x)}(\omega)\right) \\
& =\frac{\sigma_{\eta}{ }^{2}}{(2 \pi)^{d} 2^{2 \nu-1} \Gamma(2 \nu)}\left(\frac{\|\mathbf{L}\|}{\left|P_{\mathbf{h}}(\omega, \boldsymbol{\psi})\right|}\right)^{2 \nu-\frac{d}{2}} K_{2 \nu-\frac{d}{2}}\left(\|\mathbf{L}\|\left|P_{\mathbf{h}}(\omega, \boldsymbol{\psi})\right|\right) .
\end{aligned}
$$

where $\mathbf{s}(\mathbf{L})=\mathbf{s}+\mathbf{L}$, and $K_{\nu}(x)$ is the modified Bessel function of the second kind of order $\nu$. We note that in view of spatial stationarity, the right hand side expression does not depend on $\mathbf{s}$, and depends only on the Euclidean spatial distances $\|\mathbf{L}\|$ and $\|\mathbf{h}\|$. Further, as $\|\mathbf{L}\| \rightarrow 0$, the temporal spectrum of the intrinsic process $\left\{X_{t}^{\mathbf{h}}(s)\right\}$ is given by

$$
g_{0}^{(\mathbf{h})}(\omega)=\operatorname{Var}\left(J_{\mathbf{s}, \mathbf{s}+\mathbf{h}}^{(x)}(\omega)\right)=\frac{\sigma_{\eta}{ }^{2}}{(2 \pi)^{\frac{d}{2}} 2^{\frac{d}{2}}\left(\left|P_{\mathbf{h}}(\omega, \boldsymbol{\psi})\right|^{2}\right)^{2 v-\frac{d}{2}}} \frac{\Gamma\left(2 v-\frac{d}{2}\right)}{\Gamma(2 v)} .
$$

Proof. The proof is similar to the proof of Theorem 1 of the paper by Subba Rao and Terdik (2015) and hence omitted.

We note from the expression (12) for the space-time spectrum corresponding to the process satisfying the model (11), that it corresponds to a non-separable process , defined earlier. We also note further that as pointed out by one reviewer, that the assumption that the random process $\left\{\eta_{t}(\mathbf{s})\right\}$ is a white noise process in spatial coordinate $\mathbf{s}$ is a fiction, but still this assumption made in the literature. More over both covariance function, and the variance given above depend 
on $\mathbf{h}$ since the polynomial $P_{\mathbf{h}}(\omega, \boldsymbol{\psi})$ is related to the second order spectral density function of the intrinsic process $X_{t}^{\mathbf{h}}(\mathbf{s})$. We note that $g_{0}^{(\mathbf{h})}(\omega)$ depends on some parameters, say, $\psi$. We denote this function by $g_{0}^{(\mathbf{h})}(\omega, \psi)$.

Proposition 4 Let $d=2, \quad \nu=1$ and assume $\mathbf{h}$ is fixed. Then

$$
g_{0}^{(\mathbf{h})}(\omega, \psi)=\frac{\sigma_{\eta}^{2}}{4 \pi}\left|P_{\mathbf{h}}(\omega, \boldsymbol{\psi})\right|^{-2}
$$

The above result shows that the function $\left|P_{\mathbf{h}}(\omega, \boldsymbol{\psi})\right|^{2}$ is related to the stationary temporal spectrum of the process $\left\{X_{t}^{\mathbf{h}}(\mathbf{s})\right\}$. We note further that $f_{X}^{h}(\boldsymbol{\lambda}, \omega)$ is the spatio-temporal spectrum and $g_{0}^{(\mathbf{h})}(\omega, \psi)$ is the stationary temporal spectrum of the process $\left\{X_{t}^{\mathbf{h}}(\mathbf{s})\right\}$. For large $n$ and for a fixed $\mathbf{h}, \operatorname{Var}\left(J_{\mathbf{s}, \mathbf{s}+\mathbf{h}}^{(x)}(\omega)\right) \approx$ $g_{0}^{(\mathbf{h})}(\omega, \psi), \quad|\omega| \leq \pi$. Once again we note that the spectral density function $f_{X}^{(\mathbf{h})}(\boldsymbol{\lambda}, \omega)$ is non-separable.

In the above, we have shown that we can obtain a parametric expression, in a close form, for the spectral density function of the intrinsic process. The spectral density function is given by $g_{0}^{(\mathbf{h})}(\omega, \psi)$. In the following section, we consider the estimation of parameter vector $\underline{\psi}$ using the discrete Fourier transform of the $\operatorname{process}\left\{X_{t}^{\mathbf{h}}(\mathbf{s})\right\}$.

\section{Estimation of the parameters of the frequency variogram of the intrinsic process.}

Matheron (1963), Cressie (1993), Stein (2012), Yu et al. (2007), and many others have stressed the importance of the variogram in Kriging and in view of this, several methods of estimation of the variogram in the case of spatial processes have been proposed. Yu et al. (2007) have proposed nonparametric estimation of the variogram, Huang et al. (2011) proposed the estimation of the variogram and its spectrum. If one assumes that the intrinsic process satisfies a specific model which have parameters which are usually unknown, then one needs to estimate the parameters of the model. In section 7 , we have obtained an expression for the spectral density function of the intrinsic process assuming that the process satisfies the model given in Theorem 2 and we have seen that the spectrum depends on the parameter vector $\psi$. We consider the estimation of the parameter vector $\psi$ from the data $\left\{X_{t}^{\mathbf{h}}(\mathbf{s})\right\}$.

Our object here is to estimate $\boldsymbol{\psi}$ of $g_{0}^{(\mathbf{h})}(\omega, \boldsymbol{\psi})$ given the discrete Fourier Transforms $\left\{J_{\mathbf{s}, \mathbf{s}+\mathbf{h}}^{(x)}\left(\omega_{k}\right) ; i=1,2 \ldots m ; k=1,2 \ldots\left[\frac{n}{2}\right]\right.$ obtained from the intrinsic processes $\left\{X_{t}^{\mathbf{h}}\left(\mathbf{s}_{i}\right) ; t=1,2, \ldots, n ; i=1,2, \ldots m\right\}$. Let the set $N(\mathbf{h})=\left\{\mathbf{s}_{i} ; i=1,2, \ldots, m\right.$, 
$\left.\mathbf{s}_{i}, \mathbf{s}_{i}+\mathbf{h} \in \Omega\right\}$. If we are assuming that the DFT of the intrinsic process satisfies the model (11) stated in the Theorem 2, then the parameters we have to consider for the estimation are $\boldsymbol{\psi}$ of the Polynomial $P_{\mathbf{h}}(\omega, \boldsymbol{\psi})$ related to the temporal spectrum $g_{0}{ }^{(\mathbf{h})}(\omega, \psi)$ of the process $\left\{X_{t}^{\mathbf{h}}\left(\mathbf{s}_{i}\right)\right\}$. Here we obtain the likelihood function using the DFT's, and the approach is similar to the method described in Subba Rao et al. (2014). We refer to the above paper for details.

Consider the Discrete Fourier Transforms $\left.\left\{J_{\mathbf{s}_{i}, \mathbf{s}_{i}+\mathbf{h}}^{(x)}\left(\omega_{k}\right)\right)\right\}$ corresponding to the time series $\left\{Y_{t}\left(\mathbf{s}_{i}\right)\right\},\left\{Y_{t}\left(\mathbf{s}_{i}+\mathbf{h}\right)\right\}$. We note that for large $n$, the complex valued random variable $J_{\mathbf{s}_{i}, \mathbf{s}_{i}+\mathbf{h}}^{(x)}\left(\omega_{k}\right)$ is asymptotically distributed as complex normal with mean zero and variance $g_{0}{ }^{(\mathbf{h})}\left(\omega_{k}, \boldsymbol{\psi}\right)$ (see Brillinger (2001) and Giraitis et al. (2012)) and independent over distinct frequencies.. Let $M=\left[\frac{n}{2}\right]$. Consider the $M$ dimensional complex valued random vector

$$
L_{\|\mathbf{h}\|}(\omega)=\left\{J_{\mathbf{s}_{i}, \mathbf{s}_{i}+\mathbf{h}}^{(x)}\left(\omega_{1}\right), J_{\mathbf{s}_{i}, \mathbf{s}_{i}+\mathbf{h}}^{(x)}\left(\omega_{2}\right), \ldots, J_{\mathbf{s}_{i}, \mathbf{s}_{i}+\mathbf{h}}^{(x)}\left(\omega_{M}\right)\right\},
$$

which is distributed asymptotically as complex multivariate normal with mean zero and variance covariance matrix with diagonal elements

$$
\left[g_{0}^{\|\mathbf{h}\|}\left(\omega_{1}, \boldsymbol{\psi}\right), g_{0}^{\|\mathbf{h}\|}\left(\omega_{2}, \boldsymbol{\psi}\right), \ldots, g_{0}^{\|\mathbf{h}\|}\left(\omega_{M}, \boldsymbol{\psi}\right)\right] .
$$

We note that off diagonal elements of the covariance matrix are zero. Proceeding as in Subba Rao et al. (2014), we can show that the log likelihood function $l\left(\psi / J_{\mathbf{s}, \mathbf{s}+\mathbf{h}}(\omega)\right)$ is proportional to

$$
Q_{n, i}^{(\mathbf{h})}(\boldsymbol{\psi})=\sum_{k=1}^{M}\left[\ln g_{0}\|\mathbf{h}\|\left(\omega_{k}, \boldsymbol{\psi}\right)+\frac{I_{\mathbf{s}_{i}, \mathbf{s}_{i+\mathbf{h}}}^{x}\left(\omega_{k}\right)}{g_{0}(\mathbf{h})\left(\omega_{k}, \psi\right)}\right] .
$$

Now consider all the locations $\left(\mathbf{s}_{i}, \mathbf{s}_{i}+\mathbf{h}\right) ; i=1,2, \ldots, m$ belonging to the set $N(\mathbf{h})$. Then we have the pooled criterion

$$
Q_{n, N(\mathbf{h})}(\boldsymbol{\psi})=\frac{1}{|N(\mathbf{h})|} \sum_{\left(\mathbf{s}_{i}, \mathbf{s}_{i} \in N(\mathbf{h})\right)} Q_{n, i}^{(\mathbf{h})}(\boldsymbol{\psi}) .
$$

Suppose we have $H$ spatial distances $\{\mathbf{h}(l) ; l=1,2, \ldots H\}$ for which the intrinsic stationarity condition is satisfied then we can define an over all measure for minimization,

$$
Q_{n}(\boldsymbol{\psi})=\frac{1}{H} \sum Q_{n, N\left(\mathbf{h}_{l}\right)}(\boldsymbol{\psi})
$$

We minimize (17) with respect to $\boldsymbol{\psi}$. The asymptotic normality of the estimator of $\boldsymbol{\psi}$ can be proved using the methodology described in Subba Rao et al. (2014). For large $n$, we can show

$$
\sqrt{n}(\tilde{\boldsymbol{\psi}}-\boldsymbol{\psi}) \stackrel{D}{\rightarrow} N\left(0,\left[\nabla^{2} Q_{n}(\boldsymbol{\psi})\right]^{-1} V\left[\nabla^{2} Q_{n}(\boldsymbol{\psi})\right]\right),
$$


where $V=\lim _{n \rightarrow \infty} \operatorname{Var}\left[\frac{1}{\sqrt{n}} \nabla Q_{n},(\boldsymbol{\psi})\right]$, and $\nabla Q_{n}(\boldsymbol{\psi})$ is a Jacobian vector of first order partial derivatives, and $\left[\nabla^{2} Q_{n}(\boldsymbol{\psi})\right]$ is a Hessian matrix of second order partial derivatives.

\section{Test for independence of $m$ spatial time series.}

So far we have considered the analysis of spatio-temporal data using various frequency domain methods. We assumed that there is a second order dependence in space and time. It is important to test for Independence over space and time before modelling the data. Henebry (1995) proposed a test statistic for testing spatio temporal independence; and the test proposed is as an extension of Moran's test. In their book Cressie and Wikle (2011) briefly discussed the test. In this section, we propose a test for spatial independence using the Discrete Fourier Transforms and the test is based on the test proposed by Wahba (1971) which is an extension of the classical test for independence used in multivariate analysis. Here we briefly describe the test. Let.

$$
\underline{Y}_{t}^{\prime}=\left(Y_{t}\left(\mathbf{s}_{1}\right), Y_{t}\left(\mathbf{s}_{2}\right), \ldots, Y_{t}\left(\mathbf{s}_{m}\right)\right) .
$$

We say, the multivariate time series $\left\{\underline{Y}_{t}\right\}$ is second order stationary if (see Brockwell and Davis (1987))

1. $E\left(\underline{Y}_{t}\right)=\underline{\mu}$,

2. $E\left(\underline{Y}_{t}-\underline{\mu}\right)\left(\underline{Y}_{t+p}-\underline{\mu}\right)^{\prime}=\underline{\Gamma}(p)$, where

$$
\begin{aligned}
\underline{\mu}^{\prime} & =\left(\mu_{1}, \mu_{2}, \ldots, \mu_{m}\right), \\
\Gamma(p) & =\left(\sigma_{i j}(p)\right), \\
\sigma_{i j}(p) & =E\left(Y_{t}\left(\mathbf{s}_{i}\right)-\mu_{i}\right)\left(Y_{t+p}\left(\mathbf{s}_{j}\right)-\mu_{j}\right),(i, j=1,2, \ldots, m), \\
\sigma_{i j}(p) & =\sigma_{j i}(-p) .
\end{aligned}
$$

Here we are assuming that the spatio-temporal data is temporally stationary only and no assumption of spatial stationarity is assumed. We assume further that $\underline{Y}_{t}$ is Gaussian. Define the complex valued random vector

$$
\underline{J}^{\prime}\left(\omega_{k}\right)=\left(J_{\mathbf{s}_{1}}^{y}\left(\omega_{k}\right), J_{\mathbf{s}_{2}}^{y}\left(\omega_{k}\right), \ldots, J_{\mathbf{s}_{m}}^{y}\left(\omega_{k}\right)\right),
$$

where $J_{\mathbf{s}_{i}}^{y}\left(\omega_{k}\right)$ is the DFT of the time series data $\left\{Y_{t}\left(\mathbf{s}_{i}\right)\right\}$, and $\omega_{k}=\frac{2 \pi k}{n},(k=$ $\left., 1, \ldots,\left[\frac{n}{2}\right]\right)$. We know that the random vector $\underline{J}\left(\omega_{k}\right)$ is distributed as complex normal with mean $\mathbf{0}$ and variance covariance matrix $\underline{F}\left(\omega_{k}\right)$, where $\underline{F}\left(\omega_{k}\right)=\left[E\left(J_{\mathbf{s}_{i}}^{y}\left(\omega_{k}\right) J_{\mathbf{s}_{j}}^{y *}\left(\omega_{k}\right)\right]\right.$. We note that $\underline{F}\left(\omega_{k}\right)$ is a Hermitian matrix, with elements

$$
f_{\mathbf{s}_{i}, \mathbf{s}_{j}}\left(\omega_{k}\right)=E\left(J_{\mathbf{s}_{i}}^{y}\left(\omega_{k}\right) J_{\mathbf{s}_{j}}^{y *}\left(\omega_{k}\right)\right)=f_{\mathbf{s}_{j}, \mathbf{s}_{i}}\left(-\omega_{k}\right) .
$$


In the above $f_{\mathbf{s}_{i}, \mathbf{s}_{i}}\left(\omega_{k}\right)$ is the second order spectral density function of the process $\left\{Y_{t}\left(\mathbf{s}_{i}\right)\right\}$, and $f_{\mathbf{s}_{i}, \mathbf{s}_{j}}\left(\omega_{k}\right)$ is the cross spectral density function of the process $\left\{Y_{t}\left(\mathbf{s}_{i}\right)\right\}$ and $\left\{Y_{t}\left(\mathbf{s}_{j}\right)\right\}$. The cross spectral density function is usually a complex valued function.

If we assume that the spatio-temporal process $\left\{Y_{t}(\mathbf{s})\right\}$ is stationary in space and time, and further assume that the process is isotropic in space, then

$$
\begin{aligned}
& f_{\mathbf{s}_{i}, \mathbf{s}_{i}}(\omega)=f_{0}(\omega), \\
& f_{\mathbf{s}_{i}, \mathbf{s}_{j}}(\omega)=f_{\left\|\mathbf{s}_{i}-\mathbf{s}_{j}\right\|}(\omega) .
\end{aligned}
$$

In this case the matrix $\underline{F}(\omega)$ is real and symmetric, and all the diagonal elements are equal to $f_{0}(\omega)$.

As pointed out earlier, for testing spatial independence we do not need the assumption of spatial stationarity. Below we assume that the process is Gaussian. Under the null hypothesis that the spatial process is spatially independent, the spectral matrix $F(\omega)$ is a diagonal matrix for all $|\omega| \leq \pi$. For constructing the test, we proceed as in Wahba (1971). Consider the discrete Fourier transforms defined earlier. For each location $s_{i}$, let the Fourier transform be given by $\left(J_{s_{i}}^{y}\left(\omega_{l}\right)\right)$ where $\omega_{l}=\frac{2 \pi j_{l}}{n}, j_{l}=(l-1)(2 k+1)+(k+1) ; l=1,2, \ldots, M_{1}$ where $M_{1}$ is chosen such that $2(k+1) M_{1}=\frac{n-1}{2}$. (Here we assume that the number of observations n, is odd.) As in Wahba (1971) we define the cross spectral estimator of $f_{s_{i}, \mathbf{s}_{j}}(\omega)$ by

$$
\hat{f}_{s_{i}, \mathbf{s}_{j}}\left(\omega_{l}\right)=\frac{1}{2 k+1} \sum_{j_{1}=-k}^{k} I_{i, j}\left(\omega_{l}+\frac{2 \pi j_{1}}{n}\right), \quad\left(l=1,2, \ldots, M_{1}\right),
$$

where the cross periodogram $I_{i j}\left(\omega_{l}\right)=J_{s_{i}}^{y}\left(\omega_{l}\right) J_{\mathbf{s}_{j}}^{y *}\left(\omega_{l}\right)$.

Let $\hat{F}\left(\omega_{l}\right)=\left(\hat{f}_{s_{i}, \mathbf{s}_{j}}\left(\omega_{l}\right)\right) \quad\left(l=1,2, \ldots, M_{1}\right)$.

We note that the random matrices $\hat{F}\left(\omega_{l}\right) ; l=1,2, \ldots, M_{1}$, for large $k$, are approximately distributed as random matrices $\tilde{F}\left(\omega_{l}\right),(l=1,2, \ldots, M)$ which are distributed as complex Wishart, usually denoted by $W_{c}(F, m, 2 k+1)$. Wahba (1971) has shown that the likelihood ratio test for testing the null hypothesis that the matrices $F\left(w_{l}\right)$ are diagonal for all $\left\{\omega_{l}\right\}$ leads to the test statistic, for each $w_{l}$,

$$
\tilde{\boldsymbol{\lambda}}_{l}=\frac{\left|\tilde{F}\left(\omega_{l}\right)\right|}{\prod_{j=1}^{m} \tilde{f}_{\mathbf{s}_{j}, \mathbf{s}_{j}}\left(\omega_{l}\right)} \quad\left(l=1,2, \ldots, M_{1}\right),
$$

and the over-all test statistic to consider is $\Lambda=-\frac{1}{M_{1}} \sum \ln \tilde{\boldsymbol{\lambda}}_{l}$. For large $k$ and $M_{1}$, under the null hypothesis, the statistic $\boldsymbol{\Lambda}$ is asymptotically distributed as normal with mean

$$
E(\boldsymbol{\Lambda})=\sum_{j=1}^{m-1} \frac{m-j}{k^{\prime}-j}
$$


and variance

$$
\operatorname{Var}(\boldsymbol{\Lambda})=\frac{1}{M_{1}} \sum_{j=1}^{m-1} \frac{m-j}{\left(k^{\prime}-j\right)^{2}}
$$

where $k^{\prime}=2 k+1$. Under the null hypotheses of spatial independence, for large $k$ and $M$, the statistic $S=\frac{\boldsymbol{\Lambda}-E(\boldsymbol{\Lambda})}{\sqrt{\operatorname{Var}(\boldsymbol{\Lambda})}}$ is distributed as standard normal. We note that if for each $\mathbf{s}_{i},\left\{Y_{t}\left(\mathbf{s}_{i}\right)\right\}$ is a Gaussian white noise, then the spectral density function is given by $f_{\mathbf{s}_{i}, \mathbf{s}_{i}}(\omega)=\frac{\sigma_{s_{i}}{ }^{2}}{2 \pi}$, where ${\sigma_{\mathbf{s}_{i}}}^{2}$ is the variance of the white noise. If the null hypothesis is both spatially and temporally independent then the diagonal elements of the matrix $F\left(\omega_{l}\right)$ will be proportional to $\left({\sigma_{\mathbf{s}_{1}}}^{2},{\sigma_{\mathbf{s}_{2}}}^{2}, \sigma_{\mathbf{s}_{3}}{ }^{2}, \ldots, \sigma_{\mathbf{s}_{m}}{ }^{2}\right)$, and all off diagonal elements will be zero.

\section{Appendix: Discrete Fourier Transforms.}

In this section, we will briefly summarize some results related to the Discrete Fourier Transforms, further details, we refer to Subba Rao and Terdik (2015)., Brillinger (2001), Giraitis et al. (2012).

Let $\left\{Y_{t}(\mathbf{s})\right\}$, where $\left\{\mathbf{s} \in \mathbb{R}^{d} ; t \in Z\right\}$ denote a zero mean second order spatially,temporally stationary process with spectral representation

$$
Y_{t}(\mathbf{s})=\int_{-\infty}^{\infty} \int_{-\pi}^{\pi} e^{i(\mathbf{s} \cdot \boldsymbol{\lambda}+t \omega)} d Z_{y}(\boldsymbol{\lambda}, \omega)
$$

and let $\left.\left\{Y_{t}\left(\mathbf{s}_{\mathbf{i}}\right)\right) ; i=1,2, \ldots, m ; t=1,2, \ldots, n\right\}$ be a sample from the process $\left\{Y_{t}(\mathbf{s})\right\}$. We note that $Z_{y}(\boldsymbol{\lambda}, \omega)$ is a zero mean complex valued function with orthogonal increments and

$$
\begin{aligned}
E\left[d Z_{y}(\boldsymbol{\lambda}, \omega)\right] & =0, \\
E\left|d Z_{y}(\boldsymbol{\lambda}, \omega)\right|^{2} & =d F_{y}(\boldsymbol{\lambda}, \omega),
\end{aligned}
$$

where $d F_{y}(\boldsymbol{\lambda}, \omega)$ is a spectral measure, Let $d F_{y}(\boldsymbol{\lambda}, \omega)=f_{y}(\boldsymbol{\lambda}, \omega) d \boldsymbol{\lambda} d \omega$, where $f_{y}(\boldsymbol{\lambda}, \omega)$ is the spatio-temporal spectral density function of the process $\left\{Y_{t}(\mathbf{s})\right\}$. Define the Discrete Fourier Transform

$$
J_{\mathbf{s}}^{y}(\omega)=\frac{1}{\sqrt{2 \pi n}} \sum_{t=1}^{n} Y_{t}(\mathbf{s}) e^{i t \omega},|\omega| \leq \pi
$$

Proposition 5 Let the spectral representation of the process $\left\{Y_{t}\left(\mathbf{s}_{i}\right)\right\}$ be given by 18, and let $J_{\mathbf{s}}(\omega)$ be the DFT of the sample $\left\{Y_{t}(\mathbf{s}) ; t=1,2, \ldots n\right\}$. Then we have

$$
\text { 1. } Y_{t}(\mathbf{s})=\sqrt{\frac{n}{2 \pi}} \int J_{\mathbf{s}}^{y}(\omega) e^{i t \omega} d \omega
$$


2. $J_{\mathbf{s}}^{y}(\omega) \approx \int e^{i s \boldsymbol{\lambda}} \sqrt{\frac{n}{2 \pi}} d Z_{y}(\boldsymbol{\lambda}, \omega)$.

Proof. By substitution and using the properties of Dirac Delta function, one can show (2). (11) follows by inversion of (19). For details, refer to Subba Rao and Terdik (2015).

Let $I_{s}^{y}\left(\omega_{k}\right)=\left|J_{s}^{y}\left(\omega_{k}\right)\right|^{2}$ be the periodogram. The following results are well known (Priestley (1981), Brillinger (2001))

1. $E\left(I_{\mathbf{s}}^{y}\left(\omega_{k}\right)\right)=g_{\mathbf{s}}^{y}\left(\omega_{k}\right)+O\left(n^{-1}\right)$

2. $\operatorname{Var}\left(I_{\mathbf{s}}^{y}\left(\omega_{k}\right)\right)=g_{\mathbf{s}}^{y 2}\left(\omega_{k}\right)+O\left(n^{-1}\right), \quad \omega_{k} \neq 0, \pi$,

3. $\operatorname{Cov}\left(I_{\mathbf{s}}^{y}\left(\omega_{k}\right), I_{\mathbf{s}}^{y}\left(\omega_{l}\right)=O\left(n^{-1}\right) \quad\right.$ if $\quad \omega_{k}+\omega_{l} \neq 0 \quad(\bmod 2 \pi)$, In view of spatial stationarity, $g_{\mathbf{s}}^{y}(\omega)=g_{0}^{y}(\omega)$ for all $\mathbf{s}$, and

$$
g_{\mathbf{s}}^{y}(\omega)=\frac{1}{2 \pi} \sum_{k} \operatorname{Cov}\left(Y_{t}(\mathbf{s}), Y_{t+k}(\mathbf{s})\right) e^{-i \omega k},|\omega| \leq \pi
$$

4. $\operatorname{Cov}\left(J_{\mathbf{s}_{i}}^{y}\left(\omega_{k}\right), J_{\mathbf{s}_{j}}^{y}\left(\omega_{k}\right)\right)=O\left(n^{-1}\right), \quad$ if $\quad \omega_{k}+\omega_{l} \neq 0 \quad(\bmod \quad 2 \pi)$

5. $\operatorname{Cov}\left(J_{\mathbf{s}_{i}}^{y}\left(\omega_{k}\right), J_{\mathbf{s}_{j}}^{y}\left(\omega_{k}\right)\right)=\frac{1}{2 \pi} \sum_{n=-\infty}^{\infty} c\left(\mathbf{s}_{i}-\mathbf{s}_{j}, n\right) e^{-i n \omega_{k}}=g_{\mathbf{s}_{i}-\mathbf{s}_{j}}\left(\omega_{k}\right)+O\left(n^{-1}\right)$.If the process is isotropic then the spectral density function $g_{\mathbf{s}_{i}-\mathbf{s}_{j}}\left(\omega_{k}\right)=g_{\left\|\mathbf{s}_{i}-\mathbf{s}_{j}\right\|}\left(\omega_{k}\right)$ which is a real valued function. .

Acknowledgements. Part of the research reported in this paper was done when one of the authors (Subba Rao) was visiting the CRRAO AIMSCS, University of Hyderabad Campus, India which was funded by a grant from the Department of Science and Technology, Government of India (grant no. SR/S4/516/07). Also,we would like to thank Professor Noel Cressie for bringing to our attention the paper of Henebry (1995), and also for his comments on an earlier paper which lead to some results given in this paper. We would like to thank Professor Liudas Giraitis, Queen Mary University, London and Dr Suhasini Subba Rao, Texas A\&M University for reading the paper and for making many helpful comments. We would like to thank the two reviewers for many suggestions which improved the presentation.

\section{References}

D. R. Brillinger. Time Series; Data Analysis and Theory. Society for Industrial and Applied Mathematics (SIAM), Philadelphia, PA, 2001. ISBN 0-89871-501-6. Reprint of the 1981 edition. 
P. J. Brockwell and R. A. Davis. Time Series: Theory and Methods. SpringerVerlag, New York, 1987.

N. Cressie. Statistics for spatial data. Wiley series in Probability and Mathematical statistics, 1993.

N. Cressie and H.-C. Huang. Classes of nonseparable, spatio-temporal stationary covariance functions. Journal of the American Statistical Association, 94(448): 1330-1339, 1999.

N. Cressie and C. K. Wikle. Statistics for Spatio-Temporal Data. Wiley Series in Probability and Statistics, 2011.

R. S. Deo and W. W. Chen. On the integral of the squared periodogram. Stochastic processes and their applications, 85(1):159-176, 2000.

L. Giraitis, H. L. Koul, and D. Surgailis. Large sample inference for long memory processes, volume 201/2. World Scientific, 2012.

T. Gneiting. Nonseparable, stationary covariance functions for space-time data. Journal of the American Statistical Association, 97(458):590-600, 2002.

T. Gneiting, Z. Sasvári, and M. Schlather. Analogies and correspondences between variograms and covariance functions. Advances in Applied Probability, 33(3): 617-630, 2001.

E. Gringarten and C. V. Deutsch. Teacher's aide variogram interpretation and modeling. Mathematical Geology, 33(4):507-534, 2001.

E. J. Hannan. The asymptotic theory of linear time series models. J. Applied Proability, 10:130-145, 1973.

G. M. Henebry. Spatial model error analysis using autocorrelation indices. Ecological Modelling, 82(1):75-91, 1995.

C. Huang, T. Hsing, and N. Cressie. Nonparametric estimation of the variogram and its spectrum. Biometrika, 98(4):775-789, 2011.

B. Li, M. G. Genton, and M. Sherman. A nonparametric assessment of properties of space-time covariance functions. Journal of the American Statistical Association, 102(478):736-744, 2007.

C. Ma. Spatio-temporal variograms and covariance models. Advances in Applied Probability, 37(3):706-725, 2005. 
G. Matheron. Principles of geostatistics. Economic geology, 58(8):1246-1266, 1963.

M. B. Priestley. Spectral Analysis and Time Series. Academic Press, New York, 1981.

O. Schabenberger and C. A. Gotway. Statistical methods for spatial data analysis. CRC Press, 2005.

M. Sherman. Spatial statistics and Spatio-temporal data covariance function and directional statistics. John-Wiley, UK, 2011.

M. L. Stein. Interpolation of spatial data: some theory for kriging. Springer Science \& Business Media, 2012.

M. L. Stein. Space-time covariance functions. Journal of the American Statistical Association, 100(469):310-321, 2005a.

M. L. Stein. Statistical methods for regular monitoring data. Journal of the Royal Statistical Society: Series B (Statistical Methodology), 67(5):667-687, 2005b.

T. Subba Rao and Gy. Terdik. A space-time covariance function for spatiotemporal random processes and spatio-temporal prediction (kriging). ArXiv e-prints, 311.1981v2 [math.ST], 2015. (submited for publication).

T. Subba Rao, S. Das, and G. Boshnakov. A frequency domain approach for the estimation of parameters of spatio-temporal random processes. Journal of Time Series Analysis, 35:357-377, 2014.

M. Taniguchi. On estimation of the integrals of certain functions of special density. J. Appl.prob., 17:73-83, 1980.

G. Wahba. Some tests of independence for stationary multivariate time series. Journal of the Royal Statistical Society. Series B (Methodological), pages 153166, 1971.

A. M. Yaglom. Correlation Theory of Stationary Related Random Functions. Springer-Verlag, New York, 1987.

K. Yu, J. Mateu, and E. Porcu. A kernel-based method for non parametric estimation of variogram. Statistical Neerlandica, 61(2):173-197, 2007. 